\title{
Cardiac Beta Myosin Heavy Chain Diversity in Normal and Chronically Hypertensive Baboons
}

\author{
Richard D. Henkel, ${ }^{\star}$ John L. VandeBerg, ${ }^{*}$ Robert E. Shade, ${ }^{\ddagger}$ Jean J. Leger, ${ }^{\mathbf{5}}$ and Richard A. Walsh ${ }^{\star \|}$ \\ ${ }^{*}$ Department of Genetics and ${ }^{\ddagger}$ Department of Physiology and Medicine, Southwest Foundation for Biomedical Research, San Antonio, \\ Texas 78284; §Unité de Recherches de Physiopathologie Cellulaire et Moléculaire, Institut Nationale de la Santé et de la Recherche
} Médicale, Paris, France; and "Department of Medicine, The University of Texas Health Science Center, San Antonio, Texas 78284

\begin{abstract}
We have identified two distinct $\beta$-myosin heavy chains (MHCs) present in baboon myocardium by electrophoresis in gradient pore gels and by Western blots with anti-MHC MAb. The two $\beta$-MHCs have molecular masses of 210 and $200 \mathrm{kD}$ and share several antigenic determinants including an epitope recognized by a $\beta$-MHC-specific MAb. A fivefold increase in the level of the $200-\mathrm{kD} \beta$-MHC was observed in the hypertrophied left ventricles of baboons with chronic $(5.3 \pm 0.7 \mathrm{yr})$ renal hypertension. A $60 \%$ increase $(P<0.01)$ in BP and a $100 \%$ increase $(P<0.001)$ in left ventricular mass to body weight ratio occurred in hypertensive baboons compared with normotensive animals. The $\mathrm{Ca}^{2+}$-activated myosin $\mathrm{ATPase}$ activity in hypertrophied left ventricles was decreased by $35 \%(P<0.05)$ compared with controls. Normal levels of the 200-kD MHC were detected in the right ventricles and intraventricular septa of the hypertensive animals.

These data suggest that cardiac MHCs of primates may exist in alternative molecular forms that are indistinguishable by nondenaturing gel electrophoresis and that increased concentration of a second $\beta$-MHC is associated with ventricular hypertrophy $(r=0.55)$. The functional significance and mechanisms that control the concentration of $\beta$-MHC subspecies remain to be determined.
\end{abstract}

\section{Introduction}

The heart adapts to chronic pressure overload of the left ventricle $(\mathrm{LV})^{1}$ by concentric hypertrophy. Left ventricular hypertrophy is frequently caused by hypertension and may progress to a stage where contractile function diminishes, leading to end stage heart failure. Although changes in the mechanical behavior of hypertrophied ventricular muscles are well documented, the cellular and biochemical mechanisms that determine reduced intrinsic contractile performance remain poorly defined (1).

Address correspondence to Dr. Richard D. Henkel, Southwest Foundation for Biomedical Research, P.O. Box 28147, San Antonio, TX 78284.

Received for publication 5 April 1988 and in revised form 14 November 1988 .

1. Abbreviations used in this paper: BBS, borate-buffered saline; BWT, total body weight; IVS, intraventricular septum; LV, left ventricle; MHC, myosin heavy chain; RV, right ventricle.

J. Clin. Invest.

(C) The American Society for Clinical Investigation, Inc.

$0021-9738 / 89 / 05 / 1487 / 07 \$ 2.00$

Volume 83, May 1989, 1487-1493
Mechanical force in cardiac muscle is produced by the interdigitating movement of myosin and actin filaments. The transduction of chemical energy released by the hydrolysis of ATP into mechanical energy occurs at a site in the globular head of the myosin molecule. Myosin ATPase activity is known to be a major determinant of the velocity of shortening of both cardiac and skeletal muscle (2). Native myosin exists as a hexameric molecule composed of two myosin heavy chains (MHCs) that contain the catalytic sites for ATP hydrolysis, and four lower molecular weight myosin light chains (3). In small mammals (e.g., rats and rabbits) three isoforms of myosin, designated $V_{1}, V_{2}$, and $V_{3}$ in order of decreasing electrophoretic mobility in nondenaturing gels and decreasing ATPase activity, have been identified in ventricular muscle (4). The differences in electrophoretic mobility and catalytic activity of rat cardiac myosin isoforms result from the presence of two distinct types of MHCs, designated $\alpha$ - and $\beta$-chains, that exist in the native myosin molecule either as homodimers $\left(\mathrm{V}_{3}=\beta, \beta\right.$ and $\left.\mathrm{V}_{1}=\alpha, \alpha\right)$ or as a heterodimeric molecule $\left(\mathrm{V}_{2}=\alpha, \beta\right)(1)$. During cardiac adaptation to various developmental (5), hormonal $(6)$, and hemodynamic stimuli $(7,8)$, the relative proportion of the two isoforms has been shown to change as the genes encoding the $\alpha$ - and $\beta$-MHC are differentially expressed in response to such physiological conditions. The shifts in isoform expression towards increased levels of $\mathrm{V}_{1}$ myosin in response to thyrotoxicosis (6), and conversely towards increased levels of $\mathrm{V}_{3}$ myosin during pressure overload-induced hypertrophy $(7,8)$, indicate that transcriptional regulation of MHCs is a fundamental determinant of cardiac mechanical behavior in smaller mammalian species.

In contrast to these species, larger mammals (e.g., dogs, pigs, and primates) express only a single myosin isoform orthologous to rat $\mathrm{V}_{3}$ myosin in ventricular muscle, despite similar cardiac mechanical alterations to hemodynamic and hormonal stimuli (1). In particular, during concentric ventricular hypertrophy in response to pressure overload, no change in the native electrophoretic pattern of ventricular myosin in pyrophosphate gels has been detected despite evidence for $\alpha$ - to $\beta$-MHC transitions in the atria (9-11). Thus, it appears that a transcriptional alteration of myosin structure and function occurs in ventricular myocardium from smaller but not larger mammalian species. The cellular, biochemical, and molecular regulatory processes responsible for altered function of hypertrophied myocardium in larger mammals and man remain unclear.

Accordingly, we are characterizing a more physiologically relevant model of human gradual onset pressure overload hypertrophy in chronically hypertensive ( $>5 \mathrm{yr}$ ) baboons. The resulting effects of myocardial hypertrophy on MHC gene expression were characterized in a cohort of 11 hypertensive baboons with substantial ventricular hypertrophy and com- 
pared with results from normotensive baboons maintained in a similar environment. Left ventricular performance of these animals is characterized by impaired isovolumic relaxation, depressed rates of chamber emptying and filling, and unchanged end systolic elastance (12-14).

\section{Methods}

Baboon model of cardiac hypertrophy and hypertension. An experimental model of cardiac hypertrophy and hypertension was developed in baboons (Papio sp.) using the one clip, two kidney Goldblatt or the bilateral perinephritis model (15). From an initial population of 59 animals, 30 baboons were selected on the basis of the greatest extent and duration of hypertension and magnitude of left ventricular hypertrophy as determined by $\mathbf{M}$ mode echocardiography for use in a longitudinal study. The hearts of 11 animals from this cohort were collected from planned necropsies or after natural attrition. Hearts of normotensive adult baboons from other terminal experiments conducted at the Southwest Foundation for Biomedical Research were used as control tissues for biochemical analyses. Each animal underwent a 2-3-wk period of conscious, systemic intraarterial BP monitoring before necropsy using methods previously described for this laboratory (16).

Collection and processing of tissues. Baboon hearts were obtained at necropsy within an average of $1.6( \pm 1.1 \mathrm{SD}) \mathrm{h}$ postmortem and immediately placed on wet ice. Each heart was dissected into five sections consisting of left and right atria, free wall regions of the left and right ventricles (RV), and the intraventricular septum (IVS). After removal of epicardial fat, each section of cardiac tissue was further subdivided into apex, midwall, and base regions and stored at $-85^{\circ} \mathrm{C}$.

Preparation of homogenates and purification of myosin. The preparation of homogenates was performed generally as described by Hoh et al. (4). Briefly, 50-mg samples from the midwall regions of $L V$ and RV and IVS were homogenized at $4^{\circ} \mathrm{C}$ with a tissumizer (Tekmar Co., Cincinnati, OH) in $100 \mathrm{mM}$ tetrasodium pyrophosphate, $\mathrm{pH} 8.8,5$ $\mathrm{mM} \mathrm{Na}_{4}$ EDTA, and $2 \mathrm{mM}$ 2-mercaptoethanol. The homogenates were stirred at $4^{\circ} \mathrm{C}$ for $2 \mathrm{~h}$ and centrifuged at $48,000 \mathrm{~g}$ for $2 \mathrm{~h}$. Supernatants were stored at $-20^{\circ} \mathrm{C}$ in $50 \% \mathrm{vol} / \mathrm{vol}$ glycerol in a final vol of $5 \mathrm{ml}$.

Myosin was isolated from midwall sections of myocardium from hypertensive and normal baboons in general according to the method of Pope et al. (17) with minor modifications. All purification steps were conducted in buffers containing a final concentration of $2 \mathrm{mM} 2$-mercaptoethanol, $20 \mu \mathrm{M}$ leupeptin, and 10 kallikrein inhibitor units $/ \mathrm{ml}$ of aprotinin. Aliquots were collected for analysis by SDS-PAGE at three stages during the purification process. The first aliquot was collected after initial homogenization of tissue and extraction with SDS-PAGE disruption buffer (see below); this aliquot contained the cytosolic, membrane, and most myofibrillar proteins that exist in heart muscle. The second aliquot was collected from the pellet resulting from three cycles of homogenization and centrifugation in low salt buffer (100 $\mathrm{mM} \mathrm{KCl}, 50 \mathrm{mM}$ Tris- $\mathrm{HCl}, \mathrm{pH}$ 7.5); this aliquot contained a fraction of myocardial proteins enriched for myofibrillar constituents. The third aliquot was collected from the myosin preparation obtained after the sequential extraction of insoluble material from stage 2 in $600 \mathrm{mM}$ $\mathrm{KCl}$ buffer, $50 \mathrm{mM}$ Tris- $\mathrm{HCl}, \mathrm{pH} 7.5$, centrifugation at $40,000 \mathrm{~g}$ in the presence of $5 \mathrm{mM} \mathrm{MgCl}_{2}$ and $1 \mathrm{mM} \mathrm{ATP}$, and dialysis against low salt buffer; this aliquot contained primarily myosin.

ATPase assays. Measurement of calcium-activated cardiac myosin ATPase activity was performed using the microATPase method of Henkel et al. (18). Myosin used in these assays was isolated from 1-g sections of LV from six hypertensive baboons and from five age- and sex-matched control (normotensive) animals as described above. All 11 myosin samples were purified and assayed for protein concentrations and ATPase activity as a single batch to minimize variation attributable to technical factors. Protein concentrations in individual myosin samples were determined by the method of Bradford (19) using BSA as a standard.
Native gel electrophoresis. Cardiac homogenates were also analyzed under nondenaturing conditions using the pyrophosphate buffering system initially described by Hoh et al. (4) and subsequently modified to include $2 \mathrm{mM}$ cysteine in the running buffer (20), with minor modifications. Briefly, 5-10 $\mu$ l of cardiac homogenate was subjected to electrophoresis on $5 \times 0.75-\mathrm{cm}$ tube gels containing $4 \%$ total, $3.25 \%$ cross-linker acrylamide in an electrophoresis chamber (model GE-24; Pharmacia Fine Chemicals, Piscataway, NJ) with buffer recirculation between anolyte and catholyte reservoirs. Gels were run for 48 $\mathrm{h}$ at $2^{\circ} \mathrm{C}$ with a constant voltage of $80 \mathrm{~V}$. Myosin isoforms were stained with an aqueous solution composed of $0.1 \% \mathrm{wt} / \mathrm{vol}$ Coomassie blue G-250 in 50\% vol/vol methanol and 10\% vol/vol acetic acid. After electrophoresis, proteins in the gels were fixed with a $10 \% \mathrm{wt} / \mathrm{vol}$ TCA and $5 \% \mathrm{wt} / \mathrm{vol}$ sulfosalicylic acid solution in water and subsequently destained with aqueous $7 \%$ vol/vol acetic acid.

$S D S-P A G E$. Electrophoresis of cardiac homogenates and purified myosin under denaturing conditions was conducted in $8-24 \%$ total, $1 \%$ cross-linker acrylamide gradient pore gels using the buffer system described by Laemmli (21). Homogenates extracted with pyrophosphate and aliquots from stages in the purification of myosin described above were mixed with an equal volume of disruption buffer containing $4 \% \mathrm{wt} / \mathrm{vol} \mathrm{SDS}, 4 \% \mathrm{vol} / \mathrm{vol} 2$-mercaptoethanol, $20 \% \mathrm{vol} / \mathrm{vol}$ glycerol, $0.0125 \% \mathrm{wt} / \mathrm{vol}$ bromphenol blue in $50 \mathrm{mM}$ Tris- $\mathrm{HCl}$, $\mathrm{pH} 6.7$. After mixing, samples were heated to $100^{\circ} \mathrm{C}$ for $3 \mathrm{~min}$ and centrifuged at $10,000 \mathrm{~g}$ for $2 \mathrm{~min}$. Samples were subjected to electrophoresis for 15 h at a constant voltage of $75 \mathrm{~V} / \mathrm{gel}$ in $14 \mathrm{~cm} \times 15 \mathrm{~cm} \times 1.5 \mathrm{~mm}$ gels using a vertical slab gel unit (model SE 600; Hoefer Scientific Instruments, San Francisco, CA). Gels were stained with a $0.1 \% \mathrm{wt} / \mathrm{vol}$ solution of Coomassie blue G-250 in 50\% vol/vol methanol and $10 \%$ $\mathrm{vol} / \mathrm{vol}$ acetic acid and destained in aqueous $7 \% \mathrm{vol} / \mathrm{vol}$ acetic acid. Molecular weight estimates of polypeptides in SDS-PAGE gels were derived from semilogarithmic plots of relative mobility vs. molecular weight using prestained molecular weight standards (22).

Western blots. Transfer of proteins from SDS-PAGE gels to nitrocellulose membranes $(0.2-\mu \mathrm{m}$ pores; Hoefer Scientific Instruments) was performed using the buffer system of Towbin et al. (23) in a transphor unit (model TE-42; Hoefer Scientific Instruments) with a constant current of $800 \mathrm{~mA}$ at $4^{\circ} \mathrm{C}$ for $4 \mathrm{~h}$. After transfer, nitrocellulose membranes were cut into strips and immunochemically stained with MAbs specific for MHC antigens (10, 24-26) using a biotin-avidin amplification system (27). A control MAb (MOPC-21 myeloma protein) was purchased from Sigma Chemical Co. (St. Louis, MO). The immunoperoxidase staining technique was performed by incubating nitrocellulose strips in a solution composed of $2.5 \% \mathrm{wt} / \mathrm{vol}$ powdered nonfat milk, $2.5 \%$ vol/vol liquid gelatin (Norland Products, New Brunswick, NJ), $0.001 \% \mathrm{wt} / \mathrm{vol}$ thimerosol, and $0.0001 \% \mathrm{vol} / \mathrm{vol}$ antifoam A (Sigma Chemical Co.) in borate-buffered saline (BBS) for 30 $\min$ at $23^{\circ} \mathrm{C}$ to saturate protein binding sites on the nitrocellulose. Nitrocellulose strips were subsequently reacted with MAbs for $1 \mathrm{~h}$ at $37^{\circ} \mathrm{C}$, then washed with BBS containing $0.05 \mathrm{vol} / \mathrm{vol}$ TWEEN-20 (BBS-T20) and incubated with biotinylated goat anti-mouse IgG (Vector Laboratories, Burlingame, CA) for $1 \mathrm{~h}$ at $37^{\circ} \mathrm{C}$. Strips were washed a second time with BBS-T20 and then incubated with a 1:1,000 dilution of an avidin-horseradish peroxidase conjugate (Vector Laboratories, Inc.), for $20 \mathrm{~min}$ at $23^{\circ} \mathrm{C}$. After a third wash with BBS-T20, a chromogenic substrate composed of a $0.5 \% \mathrm{vol} / \mathrm{vol}$ solution of saturated 3-amino-9-ethylcarbazole in methanol (Sigma Chemical Co.) and $0.03 \% \mathrm{vol} / \mathrm{vol} \mathrm{H}_{2} \mathrm{O}_{2}$ in methanol:BBS $(20: 80)$ was added to the nitrocellulose strips for $10 \mathrm{~min}$, after which the strips were washed with water and dried.

BBS was composed of $50 \mathrm{mM} \mathrm{H}_{3} \mathrm{BO}_{3}, 150 \mathrm{mM} \mathrm{NaCl}$, and $50 \mathrm{mM}$ $\mathrm{KCl}, \mathrm{pH}$ 7.9. Primary and secondary antibodies were diluted in the $2.5 \% \mathrm{wt} / \mathrm{vol}$ nonfat milk-liquid gelatin solution described above, whereas the avidin-horseradish peroxidase conjugate was diluted in a $0.25 \% \mathrm{wt} / \mathrm{vol}$ milk and gelatin solution.

Densitometry. Since two molecular forms of MHCs were found in baboon ventricular muscle (see results), quantitation of the relative amounts of each MHC resolved by SDS-PAGE was accomplished by 
densitometric scans of Coomassie blue G-250 stained gels using a Cliniscan densitometer (Helena Laboratories, Beaumont, TX). The relative percentages of ventricular MHC subspecies were determined as follows:

$\% \beta$-MHC subspecies

$=\frac{\text { quantity of either } 210-\text { or } 200-\mathrm{kD} \text { polypeptide }}{\text { quantity of } 210-+200-\mathrm{kD} \text { polypeptides }} \times 100$

Statistics. Differences between group mean data in hypertensive vs. control baboons were determined by unpaired $t$ tests. Testing for difference between two variances was performed using a variance ratio test (28). A $P$ value of $\leq 0.05$ was considered statistically significant. Associations between BPs, extent of left ventricular hypertrophy, and levels of $\beta$-MHC were determined by least squares linear regression using a STATGRAPHICS program (Statistical Graphics Corp., Rockville, MD).

\section{Results}

A gradual onset model of chronic renal hypertension was developed in adult baboons (Papio sp.) using a one clip, two kidney Goldblatt or bilateral perinephritis wrap procedure. The mean conscious systemic arterial BP of the experimentally treated animals increased significantly $(P<0.01)$ by $\sim 60 \%$ from a normotensive value of $83 \pm 11$ to $138 \pm 23 \mathrm{mmHg}$ (Fig. 1). The hypertensive baboons (ages 11-16) survived for an average of $1,932 \pm 252 \mathrm{~d}(5.3 \mathrm{yr})$ after treatment, which represents $\sim 30 \%$ of the untreated captive baboon life expectancy. During this time a mean increase of $100 \%(P<0.001)$ in the ratio of left ventricular mass to total body weight (BWT) occurred in 9 of the 11 animals. An increase of $11 \%(P<0.01)$ in the right ventricular mass to BWT ratio and a $10 \%(P<0.01)$ increase in the IVS mass to BWT ratio were observed in the hypertensive baboons compared with normotensive controls. The average LV/BWT ratio of hypertensive baboons was $4.99 \pm 1.53$ compared with a value of $2.54 \pm 0.26$ determined for 11 normotensive male baboons maintained in an environment similar to that of the treated animals (Fig. 1). Average values for the RV/BWT and IVS/BWT ratios in hypertensive baboons $(n=11)$ were $1.05 \pm 0.29$ and $1.48 \pm 0.47$, respectively, whereas respective values of $0.95 \pm 0.20$ and $1.34 \pm 0.29$ were observed for normotensive baboons $(n=82)$. Substantial left ventricular hypertrophy did not occur in two baboons in spite of significantly higher BPs in these animals.

A

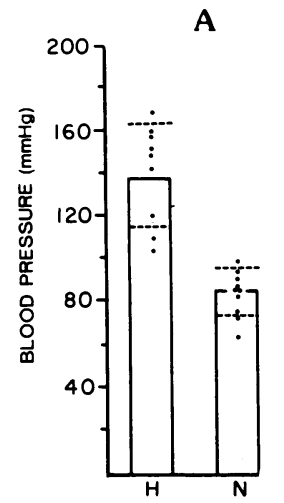

B

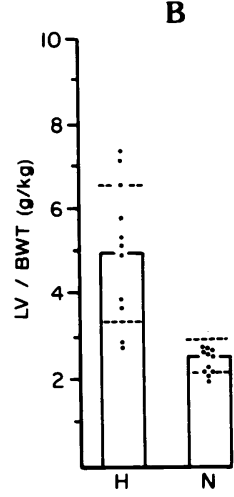

Figure 1. BPs and left ventricular hypertrophy in a baboon model of chronic renal hypertension. The mean conscious arterial BP in hypertensive $(H)$ and normotensive $(N)$ baboons were monitored over a 2-3-wk period with an intraarterial catheter $(A)$. The individual data points are shown with the group mean indicated at the top of each bar. $1 \mathrm{SD}$ above and below the mean are indicated by dashed lines. The LV/BWT ratios for both cohorts of baboons were determined at necropsy and are shown in $B$.
Analysis of native myosin isoforms was accomplished in pyrophosphate-buffered, nonrestrictive polyacrylamide gels using myosin extracted from cardiac myofibrils with $100 \mathrm{mM}$ tetrasodium pyrophosphate. As represented in Fig. 2, a single charge form of myosin was found in homogenates of LV myocardium from both hypertensive and normotensive animals. The same isoform pattern was observed in the RV and IVS from both cohorts of baboons (data not shown).

The calcium-activated ATPase activity of cardiac myosin in left ventricular myocardium from a subset of hypertensive baboons was determined for comparison with that from a group of normotensive animals. As shown in Table I, myosin ATPase activity from hypertensive baboons was determined to be $0.181 \mu \mathrm{mol}$ of inorganic phosphate released/min per $\mathrm{mg}$ myosin. This value was significantly lower $(P<0.05)$ than the average value of $0.273 \mu \mathrm{mol}$ of inorganic phosphate released/ min per mg of myosin determined for myosin ATPase activity from control animals.

Cardiac myosin from hypertensive and normal baboons was further analyzed after denaturation into heavy and light chain components by SDS-PAGE in gradient pore gels. As seen in Fig. 3, left ventricular myocardium from two hypertensive baboons (animal numbers X2296 and X3156) and one normotensive animal (X2961) contained a $210-\mathrm{kD}$ polypeptide in cardiac homogenates (sample 1), in a fraction enriched for myofibrillar proteins (sample 2), and in partially purified preparations of myosin (sample 3 ). This polypeptide was tentatively identified as MHC based on previous reports (3). An additional polypeptide with an estimated molecular mass of $200 \mathrm{kD}$ was observed at increased levels in SDS-PAGE polypeptide profiles of hypertensive baboon left ventricular myocardium. A trace amount of this $200-\mathrm{kD}$ polypeptide was found in most but not all of the LVs of control animals. The 200-kD polypeptide copurified with the $210-\mathrm{kD}$ MHC in samples collected from hypertensive animals.

The immunochemical properties of the $200-\mathrm{kD}$ polypeptide found in increased levels in hypertensive baboon LVs and the normal 210-kD MHC were examined in Western blotting experiments using MAbs that had been raised against human myosin and were specific for several distinct MHC epitopes. In these experiments, MHCs in cardiac muscle homogenized in $100 \mathrm{mM}$ tetrasodium pyrophosphate were immunoperoxidase

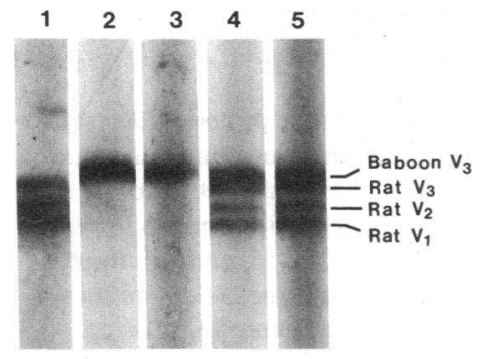

Figure 2. Native pyrophosphate gel electrophoresis of rat and baboon cardiac myosin. Electrophoresis of cardiac myosin isoforms in rat and baboon LVs was performed under nondenaturing conditions in pyrophosphate-buffered, nonrestrictive polyacrylamide gels. Lane 1 illus-

trates the typical Coomassie blue G-250 staining pattern seen for rat cardiac myosin which is composed of three isoforms designated $V_{1}$, $V_{2}$, and $V_{3}$ in order of decreasing electrophoretic mobility. The mobilities of cardiac myosin in the left ventricular muscle of normal and hypertensive baboons are shown in lanes 2 and 3, respectively. The electrophoretic profiles of mixtures of rat cardiac myosin with either normal or hypertensive baboon myosin (lanes 4 and 5 , respectively) further demonstrate the similarity between the electrophoretic mobilities of normal and hypertensive baboon cardiac myosin. 
Table I. Calcium-stimulated Myosin ATPase Activity in Hypertensive and Normotensive Baboons

\begin{tabular}{llll}
\hline Animal identification number & $\mathrm{Sex}$ & \multicolumn{2}{c}{$\mathrm{Ca}^{2+}$-activated myosin ATPase* } \\
\hline Hypertensive baboons & & & \\
X2655 & F & & 0.211 \\
X3156 & M & & 0.171 \\
X1643 & M & & 0.149 \\
X1829 & M & & 0.125 \\
X1184 & M & & 0.159 \\
X1111 & F & & 0.273 \\
& & $\bar{X}$ & $0.181^{\ddagger}$ \\
& & SD & 0.048 \\
Normotensive baboons & & & \\
X2703 & F & & 0.276 \\
X3016 & M & & 0.287 \\
X2627 & F & & 0.234 \\
X1828 & M & & 0.225 \\
X1929 & M & & 0.344 \\
& & $\bar{X}$ & 0.273 \\
& & SD & 0.042 \\
\hline
\end{tabular}

* Expressed as micromoles of inorganic phosphate released per minute per milligram of myosin.

${ }^{\ddagger} P<0.05$ vs. control.

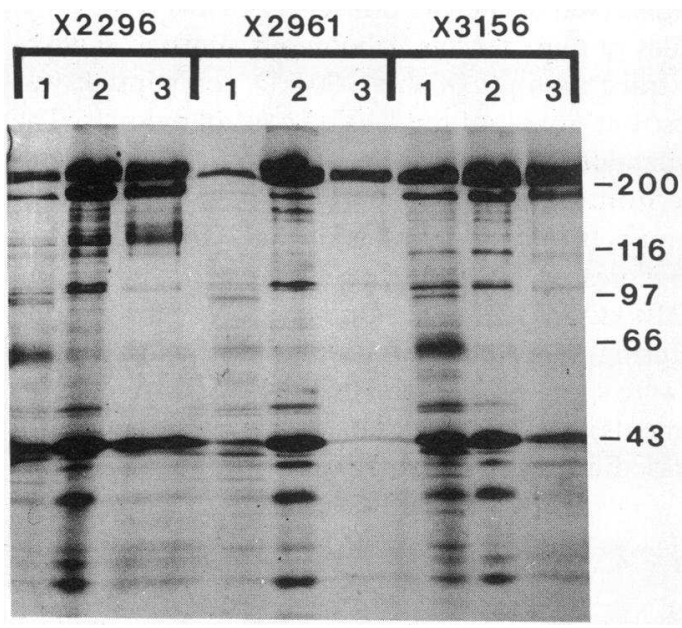

Figure 3. SDS-PAGE of baboon cardiac muscle proteins. Electrophoresis of baboon myocardial proteins was performed under denaturing conditions in gradient pore SDS-PAGE. Electrophoretic profiles of three fractions of myocardial proteins (lanes 1-3) from two hypertensive baboons (animal numbers X2296 and X3156) and a normotensive baboon (X2961) stained with Coomassie blue G-250 are shown relative to the molecular weights of standards expressed in kilodaltons. The first fraction of myocardial proteins (lane 1) containing cytosolic, membrane, and myofibrillar components was prepared by homogenization of left ventricular muscle from each animal followed by solubilization of proteins in $2 \%$ SDS. The second fraction (lane 2) of proteins was enriched for myofibrillar proteins by removal of cytosolic and membrane components. The third fraction contains primarily myosin heavy chains (200-210 kD) and actin (43 kD). This figure demonstrates that the $200-\mathrm{kD}$ polypeptide, which is expressed to a greater degree in hypertrophied ventricular myocardium, copurifies with the $210-\mathrm{kD}$ MHC. stained with MAb after separation and transfer from SDSPAGE gradient pore gels. The reactivities of four types of antibodies including an anti- $\beta$-MHC-specific MAb, an anti- $\alpha$ MHC MAb, a MAb cross-reactive with both types of MHCs, and a control MAb, are shown in Fig. 4 in Western blots with left atrial and left ventricular homogenates from a representative hypertensive baboon (X3156). The anti- $\beta$-MHC MAb reacted strongly with the $210-\mathrm{kD}$ MHCs in hypertrophied ventricle and to a lesser extent with the $210-\mathrm{kD}$ MHCs found in the atrial homogenate (lanes $V$ and $A$, respectively). The 200-kD polypeptide was also strongly stained in ventricular homogenates by the anti- $\beta$-MHC MAb as were several smaller bands in the $150-180 \mathrm{kD}$ range. The MAb specifically reactive with $\alpha$-MHCs stained only atrial MHC, producing an intense reaction with the $210-\mathrm{kD}$ atrial MHCs, while detecting trace amounts of the $200-$ and $150-\mathrm{kD}$ polypeptides. The MAb cross-reactive with both $\alpha$ - and $\beta$-MHCs strongly stained the 210-kD MHC to the same extent in both LA and LV homogenates. This antibody recognized an antigenic determinant on the $200-\mathrm{kD}$ polypeptide found in large amounts in hypertrophied LV and in trace amounts in LA as well as several of the lower molecular weight bands in the 150-180 kD range. Based on these results, we conclude that the $200-\mathrm{kD}$ polypeptide is a second molecular form of a $\beta$-type MHC, and for the sake of convenience the 210 - and $200-\mathrm{kD}$ polypeptides will be referred to subsequently as $\beta 1$ and $\beta 2$ MHCs, respectively.

Based on limited observations that several hypertensive baboons expressed higher than normal levels of the $\beta 2 \mathrm{MHC}$, we subsequently conducted an electrophoretic survey of 11 hypertensive and 11 normal baboons to quantitate levels of $\beta 2$ MHCs in a larger population. The levels of both $\beta 1$ and $\beta 2$ MHCs found in midwall sections of LV, RV, and IVS from hypertensive and normal baboons were determined by densitometry of SDS-PAGE gradient pore gels and expressed as the percentage of the total MHCs (total area under $\beta 1$ and $\beta 2$ curves in scans) found in each $\beta$-MHC band. A mean value for $\beta 2$ MHC levels of $26 \% \pm 13$ and a range of values between 5 and $51 \%$ was found in hypertrophied LVs (Fig. 5), whereas average $\beta 2$ MHC values of $<6 \% \pm 5$ were detected in all normal samples and in the RV and IVS of hypertensive animals ( $P$ $<0.001)$. Representative experimental results from the electrophoretic survey are shown in Fig. 6. SDS-PAGE polypeptide profiles from normal hearts shown in Fig. 6 include two animals (X1931 and X3300) with $\beta 2$ MHC levels representative of the normotensive cohort average and one animal with the highest and one with the lowest $\beta 2$ MHC values in the normotensive group (X2094 and X3580, respectively). Profiles shown for the hypertensive baboons include an animal with $\beta 2$ MHC levels near the mean of the hypertensive group (X3156), two animals with the highest levels of $\beta 2$ MHCs (X1829 and X1184), and the single hypertensive (X1643) animal in the group with normal levels of $\beta 2$ MHCs similar to the mean value of the normotensive group.

The associations between LV/BWT and mean arterial BP and between LV/BWT and the level of $\beta 2$ MHC were determined by least squares linear regression analysis. A correlation coefficient $(r)$ of 0.51 and a significance level of the regression $(P \leq 0.062)$ were determined for the relationship between $\mathrm{LV} /$ BWT and mean arterial BP. An $r$ value of $0.55(P \leq 0.009)$ was observed between LV/BWT and the level of $\beta 2$ MHC in baboons. Considerable variation was observed in the distribution of $\beta 2$ MHCs in the LV of hypertensive baboons. The variance 


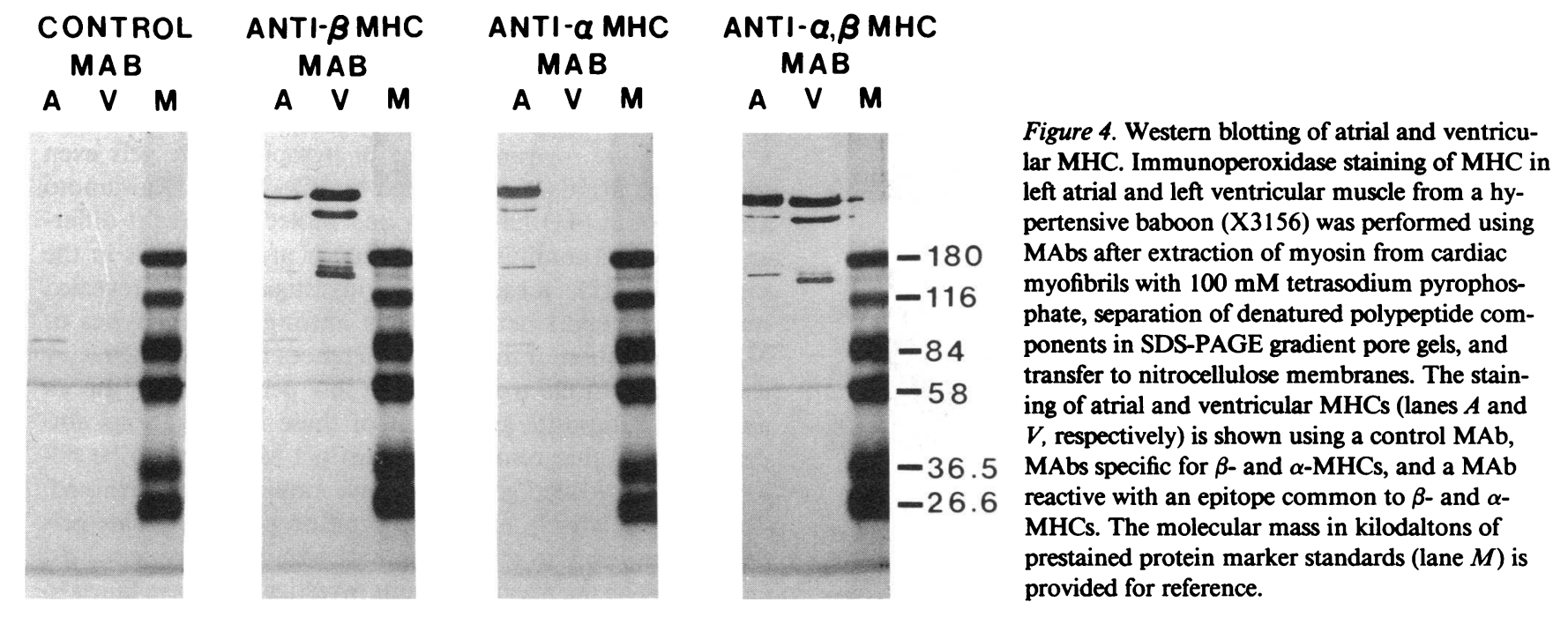

of the hypertensive group of baboons with respect to the level of $\beta$-MHCs was greater than that of the normotensive cohort $(P<0.005)$. There was also greater variability in the hypertensive baboons in the distribution of BPs $(P<0.02)$ and left ventricular hypertrophy $(P<0.0001)$.

\section{Discussion}

We have developed a nonhuman primate model of gradual onset chronic renal hypertension in which significant increases in mean conscious systemic arterial BP and left ventricular hypertrophy occur in experimental animals compared with untreated normotensive animals. Using biochemical and immunochemical techniques to analyze the cardiac myosin from the hypertensive baboons we have detected a second form of $\beta$-MHC in the ventricular myocardium by SDS-PAGE in gradient pore gels. The new form of MHC has an estimated molecular mass that is $10 \mathrm{kD}$ smaller in size than the predominant $210-\mathrm{kD}$ form of MHC usually found in myocardium. We have designated the $210-$ and $200-\mathrm{kD}$ polypeptides as $\beta 1$ and $\beta 2$ MHCs, respectively. Our identification of the smaller polypeptide as a $\beta$-type MHC is supported by the following observations: $(a)$ the $\beta 2$ MHC copurifies with the $\beta 1 \mathrm{MHC}$ due to similar solubility properties in low and high ionic strength buffers; $(b)$ both types of $\beta$-MHCs are readily extracted from myocardium with tetrasodium pyrophosphate, although only a single native myosin isoform can be detected in hypertro-

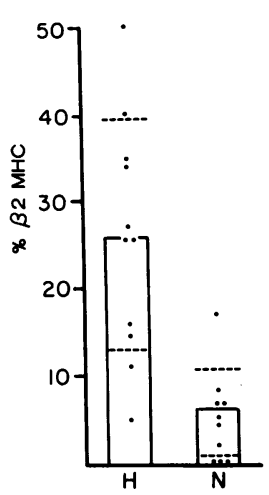

Figure 5. Levels of $\beta 2$ MHC in chronically hypertensive and normotensive baboons. The levels of $\beta 2$ MHC in the midwall region of LVs from hypertensive $(H)$ and normotensive $(N)$ baboons were determined by densitometry from SDS-PAGE gradient pore gels. Individual measurements are shown as dots with the group means indicated at the tops of the bars. $1 \mathrm{SD}$ above and below the mean are shown as dashed lines. phied myocardium by pyrophosphate gel electrophoresis; and (c) both types of $\beta$-MHCs share at least two common antigenic determinants that are recognized by MAbs in Western blots including an epitope defined by a $\beta$-MHC-specific MAb. An $\alpha$-MHC-specific MAb did not react with either $\beta$-MHC. The amount of $\beta 2 \mathrm{MHC}$ was greatly increased, and the $\mathrm{Ca}^{2+}$-activated myosin ATPase activity was decreased in the left ventricular myocardium of chronically hypertensive baboons compared with control animals.

The functional significance of increased $\beta 2$ MHC levels is unknown, although reduced myosin ATPase activity in myocardium containing high levels of the smaller MHC suggests that the $\beta 2$ MHC may be less enzymatically active than the $\beta 1$ MHC (Table I). It has been well documented that the different catalytic properties of cardiac $\alpha$ - and $\beta$-MHCs account for the disparate shortening velocities of muscles containing each type of MHC $(1,2)$. The differential expression of $\alpha$ - and $\beta$-MHCs in the hearts of small mammals and in the atria of large animals serves as a mechanism for cardiac adaptation to changes in hemodynamic load $(7,8)$, hormonal stimulation $(6)$, and developmental programs (5). The same type of adaptive mechanism, however, has not been observed in ventricular myocardium of large mammals. Although low levels of $\alpha$-MHCs have been detected in human ventricles by several investigators using immunological techniques $(29,30)$, others report the exclusive expression of $\beta$-MHCs in these tissues (31). In any case, $\beta$-type chains have been shown to be the predominant form of MHC found in ventricular muscle of large animals regardless of the physiologic environment (1).

Some evidence suggesting the existence of more than one type of $\beta$-MHC in ventricles of large mammals has been obtained using biochemical (32) and immunological techniques $(10,33)$; however, these studies did not detect differences in the molecular weights of $\beta$-MHCs such as those that we have observed in hypertensive baboon ventricles. None of the studies which have presented evidence of $\beta$-MHC heterogeneity, including our own, have determined the origin of the structural variation in these proteins. Four possible mechanisms that might produce the $\beta$-MHC subtypes include: $(a)$ encoding of the two types of $\beta$-MHCs by distinct genes; $(b)$ posttranscriptional splicing of RNA from the same gene; $(c)$ in vivo, posttranslational modification of a single $\beta$-MHC gene prod- 

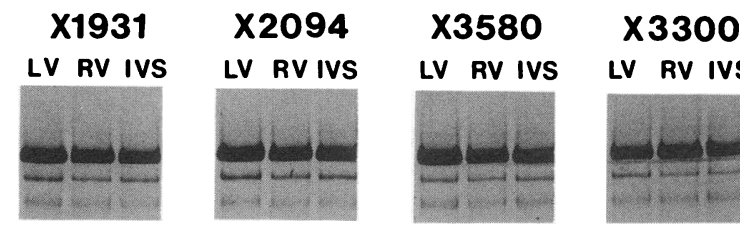

LV RV IVS
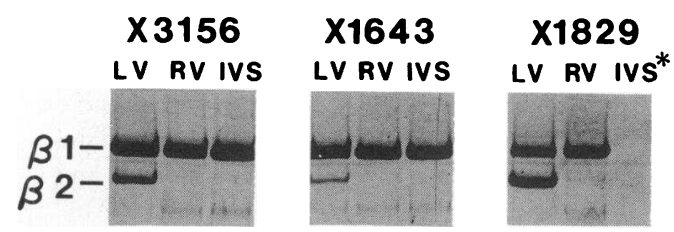

$X 1184$

LV RV IVS

Figure 6. Electrophoresis of cardiac MHC from normotensive and hypertensive baboons. Cardiac MHC were separated by SDS-PAGE in gradient pore gels after extraction from LV, RV, and IVS from normotensive (top) and hypertensive (bottom) baboon myocardium and stained with Coomassie blue G-250. Two MHCs identified as $\beta 1$ and $\beta 2$ with estimated molecular masses of 210 and $200 \mathrm{kD}$, respectively, are indicated in the hypertensive groups. Note that the IVS tissue sample for X1829 was not available for analysis by SDS-PAGE.

uct; and (d) postmortem proteolysis of a single gene product. We consider the last possibility to be the most remote for several reasons. First, the baboon hearts examined in our study were collected and processed within several hours $(1.6 \pm 1.1 \mathrm{~h})$ after cessation of blood flow. Klotz and co-workers (34) have shown that $\mathrm{Ca}^{2+}$-activated myosin ATPase in human ventricles was not altered if cardiac tissue was processed within $24 \mathrm{~h}$ after death. Second, it is unlikely that postmortem proteolysis would occur exclusively in the LV of hypertensive baboons. No significant differences were observed between the levels of $\beta 2$ MHCs found in the RV and IVS samples from hypertensive and control animals. Third, the same relative abundance of $\beta 2$ MHCs was generally found in partially purified myosin as was found in crude myosin extracts of pyrophosphate homogenized myocardium. It is unlikely that the same degree of postmortem proteolysis would be produced in the presence of protease inhibitors included in our purification buffers as would be found in the pyrophosphate solution lacking these agents. However, Siemankowski and Dreizen have previously shown that during LV hypertrophy produced by abrupt aortic banding, cardiac MHCs may be more susceptible to either proteolytic or thermally activated, heat-stable, nonenzymatic processes (35).

Although the molecular weight variation that we observed in $\beta$-MHCs from hypertrophied ventricular myocardium has not been reported previously, differences of the same magnitude between molecular weights of MHCs seen in our experiments have been detected in skeletal muscle myosins. Rushbrook and Stracher (36) reported molecular weight heterogeneity in MHCs from skeletal muscle using 5\% acrylamide SDS gels. Umeda et al. (37) established that these MHC isoforms were not the result of proteolytic processes by demonstrating the existence of distinct mRNAs coding for fast and slow MHCs in a cell-free translation system. Other studies have confirmed the existence of multiple MHC isoforms in skeletal muscle (38). Betto et al. (39) demonstrated that three types of MHCs could be resolved by SDS-PAGE from rat skeletal muscle corresponding to myosins with different muscle shortening velocities and fatigue sensitivities.
Molecular weight polymorphism in smooth muscle MHCs has also been described by several investigators, and some interesting similarities with our experimental results were reported. Rovner et al. (40) found that smooth muscle contained a single native myosin isoform in pyrophosphate gels even though two MHCs were detected by SDS-PAGE. Kawamoto and Adelstein (41) have described molecular weight differences between nonmuscle and smooth muscle MHCs in the range of 4-9 kD. In each of these investigations that revealed molecular weight heterogeneity among various types of MHCs, resolution of the slight differences in size of the heavy chains required electrophoresis in low percentage polyacrylamide or composite gels. Most of these investigations also demonstrated that resolution of distinct MHCs could be obscured in overloaded gels, as we have empirically determined. The use of relatively high concentration polyacrylamide gels and the tendency to overload samples to detect myosin light chains may be the reason previous investigations have failed to reveal MHC variation in ventricular myosin of larger mammals. Alternatively, we have not ruled out the possibility that the $\beta$-MHC variation observed in baboons might not be present in hypertrophied LVs of other larger mammalian species.

In summary, we have identified a new subtype of $\beta$-MHC in baboon myocardium that is found at increased levels in the LV of animals with chronic hypertension and substantial pressure overload left ventricular hypertrophy. The mechanism(s) that regulate expression of the $\beta 2$ MHC are unknown. We hypothesize that reduced or absent functional activity of this myosin variant may underlie the altered mechanical behavior of pressure overload ventricular hypertrophy in higher mammalian species.

\section{Acknowledgments}

We are grateful for the assistance of Dr. K. D. Carey, Dr. Claude Dechesne, Mr. David Cragg, Ms. Mary Torres, Mr. David Weaver, and Mr. Stephen Woodford.

This work was supported by grants from the National Heart, Lung and Blood Institute of the National Institutes of Health (HL-33579 and 33579-01S1).

\section{References}

1. Swynghedauw, B. 1986. Developmental and functional adaptation of contractile proteins in cardiac and skeletal muscles. Physiol. Rev. 66:710-771.

2. Barany, M. 1967. ATPase activity of myosin correlated with speed of muscle shortening. J. Gen. Physiol. 50(Suppl):197-218.

3. Harrington, W. F., and M. E. Rodgers. 1984. Myosin. Annu. Rev. Biochem. 53:35-73.

4. Hoh, J. F. Y., P. A. McGrath, and P. T. Hale. 1978. Electrophoretic analysis of multiple forms of rat cardiac myosin: effects of hypophysectomy and thyroxine replacement. J. Mol. Cell. Cardiol. 10:1053-1076.

5. Lompre, A. M., J. J. Mercadier, C. Wisnewsky, P. Bouveret, C. Pantaloni, A. D'Albis, and K. Schwartz. 1981. Species- and age-dependent changes in the relative amounts of cardiac myosin isoenzymes in mammals. Dev. Biol. 84:286-290.

6. Morkin, E. 1979. Stimulation of cardiac myosin adenosine triphosphatase in thyrotoxicosis. Circ. Res. 44:1-7.

7. Lompre, A.-M., K. Schwartz, A. d'Albis, G. Lacombe, N. V. Thiem, and B. Swynghedauw. 1979. Myosin isoenzyme redistribution in chronic heart overloading. Nature (Lond.). 282:105-107. 
8. Litten, R. Z., III, B. J. Martin, R. B. Low, and N. R. Alpert. 1982. Altered myosin isozyme patterns from pressure-overloaded and thyrotoxic hypertrophied rabbit hearts. Circ. Res. 50:856-864.

9. Gorza, L., P. Pauletto, A. C. Pessina, S. Sartore, and S. Schiaffino. 1981. Isomyosin distribution in normal and pressure-overloaded rat ventricular myocardium: an immunohistochemical study. Circ. Res. 49:1003-1009.

10. Bouvagnet, P., J. Leger, F. Pons, C. Dechesne, and J. J. Leger. 1984. Fiber types and myosin types in human atrial and ventricular myocardium: an anatomical description. Circ. Res. 55:794-804.

11. Wisenbaugh, T., P. Allen, G. Cooper IV, H. Holygrefe, G. Beller, and B. Carabello. 1983. Contractile function, myosin ATPase activity and isozymes in the hypertrophied pig left ventricle after a chronic progressive pressure overload. Circ. Res. 53:332-341.

12. Crawford, M. H., R. A. Walsh, D. Cragg, G. L. Freeman, and J. Miller. 1987. Echocardiographic left ventricular mass and function in the hypertensive baboon. Hypertension. 10:339-345.

13. Latham, R. D., B. J. Rubal, N. Westerhof, P. Sipkema, and R. A. Walsh. 1987. Nonhuman primate model for regional wave travel and reflections along aortas. Am. J. Physiol. 253:H299-H306.

14. Latham, R. D., B. J. Rubal, P. Sipkema, N. Westerhof, R. Virmani, M. Robinowitz, and R. A. Walsh. 1988. Ventricular/vascular coupling and regional arterial dynamics in the chronically hypertensive baboon: correlation with cardiovascular structural adaptation. Circ. Res. 63:798-811.

15. McGill, H. C., Jr., K. D. Carey, C. A. McMahan, Y. N. Marinez, T. E. Cooper, G. E. Mott, and C. J. Schwartz. 1985. Effects of two forms of hypertension on atherosclerosis in the hyperlipidemic baboon. Arteriosclerosis. 5:481-493.

16. Bishop, V. S., R. E. Shade, J. R. Haywood, and C. Hamm. 1987. Sinoaortic denervation in the nonhuman primate. Am. J. Physiol. 252:R294-R298.

17. Pope, B., J. F. Y. Hoh, and A. Weeds. 1980. The ATPase activities of rat cardiac myosin isoenzymes. FEBS (Fed. Eur. Biochem. Soc.) Lett. 118:205-208.

18. Henkel, R. D., J. L. VandeBerg, and R. A. Walsh. 1988. A microassay for ATPase. Anal. Biochem. 169:312-318.

19. Bradford, M. M. 1976. A rapid and sensitive method for the quantitation of microgram quantities of protein utilizing the principle of protein-dye binding. Anal. Biochem. 72:248-254.

20. Hoh, J. F. Y. 1978. Light chain distribution of chicken skeletal muscle myosin isoenzymes. FEBS (Fed. Eur. Biochem. Soc.) Lett. 90:297-300.

21. Laemmli, U. K. 1970. Cleavage of structural proteins during the assembly of the head of bacteriophage T4. Nature (Lond.). 227:680-685.

22. Shapiro, A. L., E. Vinuela, and J. V. Maizel, Jr. 1967. Molecular weight estimation of polypeptide chains by electrophoresis in SDSpolyacrylamide gels. Biochem. Biophys. Res. Commun. 28:815-820.

23. Towbin, H., T. Staehelin, and J. Gordon. 1979. Electrophoretic transfer of proteins from polyacrylamide gels to nitrocellulose sheets: procedure and some applications. Proc. Natl. Acad. Sci. USA. 76:4350-4354.

24. Bouvagnet, P., J. Leger, C. A. Dechesne, G. Dureau, M. Anoal, and J. J. Leger. 1985. Local changes in myosin types in diseased human atrial myocardium: a quantitative immunofluorescence study. Circulation. 72:272-279.
25. Dechesne, C., J. Leger, P. Bouvagnet, M. Claviez, and J. J. Leger. 1985. Fractionation and characterization of two molecular variants of myosin from adult human atrium. J. Mol. Cell. Cardiol. 17:753-767.

26. Dechesne, C. A., J. Leger, P. Bouvagnet, H. Mairhofer, and J. J. Leger. 1985. Local diversity of myosin expression in mammalian atrial muscles: variations depending on age and thyroid state in the rat and the rabbit. Circ. Res. 57:767-775.

27. Brower, M. S., C. L. Brakel, and K. Garry. 1985. Immunodetection with streptavidin-acid phosphatase complex on Western blots. Anal. Biochem. 147:382-386.

28. Zar, J. H. 1974. Biostatistical Analysis. Prentice-Hall, Inc., Englewood Cliffs, NJ. 105-107.

29. Tsuchimochi, H., M. Sugi, M. Kuro-o, S. Ueda, F. Takaku, S. Furuta, T. Shirai, and Y. Yazaki. 1984. Isozymic changes in myosin of human atrial myocardium induced by overload. Immunohistochemical study using monoclonal antibodies. J. Clin. Invest. 74:662-665.

30. Mercadier, J.-J., P. Boveret, L. Gorza, S. Schiaffino, W. A. Clark, R. Zak, B. Swynghedauw, and K. Schwarts. 1983. Myosin isozymes in normal and hypertrophied human ventricular myocardium. Circ. Res. 53:52-62.

31. Cummins, P. 1984. Contractile protein transitions in human cardiac overload: reality and limitations. Eur. Heart J. 5(Suppl F):119-127.

32. Flink, I. L., and E. Morkin. 1984. Sequence of the 20-kilodalton heavy chain peptide from the carboxyl-terminus of bovine cardiac myosin subfragment-1. J. Clin. Invest. 74:639-646.

33. Tsuchimochi, H., M. Kuro-o, H. Koyama, M. Kurabayashi, M. Sugi, F. Takaku, S. Furuta, and Y. Yazaki. 1988. Heterogeneity of $\beta$-type myosin isozymes in the human heart and regulational mechanisms in their expression. J. Clin. Invest. 81:110-118.

34. Klotz, C., M. C. Aumont, J. J. Leger, and B. Swynghedauw. 1975. Human cardiac myosin ATPase and light subunits: a comparative study. Biochim. Biophys. Acta. 386:461-469.

35. Siemankowski, R. F., and P. Dreizen. 1978. Canine cardiac myosin with special reference to pressure overload cardiac hypertrophy. I. Subunit composition. J. Biol. Chem. 253:8648-8658.

36. Rushbrook, J. I., and A. Stracher. 1979. Comparison of adult, embryonic, and dystrophic myosin heavy chains from chicken muscle by sodium dodecyl sulfate/polyacrylamide gel electrophoresis and peptide mapping. Proc. Natl. Acad. Sci. USA. 76:4331-4334.

37. Umeda, P. K., R. Zak, and M. Rabinowitz. 1980. Purification of messenger ribonucleic acids for fast and slow myosin heavy chains by indirect immunoprecipitation of polysomes from embryonic chick skeletal muscle. Biochemistry. 19:1955-1965.

38. Carraro, U., and C. Catani. 1983. A sensitive SDS-PAGE method separating myosin heavy chain isoforms of rat skeletal muscles reveals the heterogeneous nature of the embryonic myosin. Biochem. Biophys. Res. Commun. 116:793-802.

39. Betto, D. D., E. Zerbato, and R. Betto. 1986. Type 1, 2A, and 2B myosin heavy chain electrophoretic analysis of rat muscle fibers. Biochem. Biophys. Res. Commun. 138:981-987.

40. Rovner, A. S., M. M. Thompson, and R. A. Murphy. 1986. Two different heavy chains are found in smooth muscle myosin. Am. J. Physiol. 250:C861-C870.

41. Kawamoto, S., and R. S. Adelstein. 1987. Characterization of myosin heavy chains in cultured aorta smooth muscle cells: a comparative study. J. Biol. Chem. 262:7282-7288. 